\title{
A finite word poset
}

\author{
Péter L. Erdős \\ A. Rényi Institute of Mathematics \\ Hungarian Academy of Sciences, \\ Budapest, P.O. Box 127, H-1364 Hungary \\ elp@renyi.hu \\ Péter Sziklai \\ Technical and Eötvös Universities, Budapest \\ sziklai@cs.elte.hu \\ David C. Torney \\ Theoretical Biology and Biophysics, \\ Mailstop K710, Los Alamos National Laboratory, \\ Los Alamos, New Mexico, 87545, USA; \\ dct@lanl.gov *
}

Submitted: March 1, 2000; Accepted: July 26, 2000

\begin{abstract}
Our word posets have finite words of bounded length as their elements, with the words composed from a finite alphabet. Their partial ordering follows from the inclusion of a word as a subsequence of another word. The elemental combinatorial properties of such posets are established. Their automorphism groups are determined (along with similar result for the word poset studied by Burosch, Frank and Röhl [4]) and a BLYM inequality is verified (via the normalized matching property).
\end{abstract}

AMS Classification: Primary - 06A07. Secondary - 06B25, 68R15.

\section{Introduction}

Combinatorics on words (or on finite sequences) is a well developed independent theory, rooted in several branches of mathematics, such as group theory and probability, and applied in such areas as computer science and automata theory. It considers finite sequences

*This work was supported, in part, by Hungarian NSF, under contract Nos. T29255, F30737, D32817, Eötvös grant and by the U.S.D.O.E. 
from a finite alphabet, $\Gamma=\{1,2, \ldots, k\}$. These sequences form a partially ordered set (or poset for short), with the ordering following from inclusion of one sequence as a subsequence of another sequence. The Higman theorem establishes one of the most basic properties of this infinite poset: it contains no infinite antichain (Higman, 1952, [9]). An excellent introduction to this topic is due to M. Lothaire [13].

In this paper we study the finite version of this poset: let $\mathcal{P}^{(n)}$ denote the set of all sequences of lengths up to $n$-composed from the finite alphabet $\Gamma$-equipped with the aforementioned sequence-subsequence partial ordering. This poset clearly has a rank function (the rank of a sequence being its length); therefore, it is referred to as graded. We denote the $i$ th level of $\mathcal{P}^{(n)}$ by $\mathcal{P}_{i}^{(n)}$, comprising all $k^{i} i$-sequences, $0 \leq i \leq n$.

Deleting elements occurring at given positions in a sequence yields a subsequence $[3$, p. 569]. To be explicit, a subsequence retains no information about the original positions of its elements. For example, the sequence 1211 yields the distinct 2-subsequences 11, 12, and 21. Subsequences are elsewhere also called subwords, but we use only the notion of subsequences in the sequel.

The posets $\mathcal{P}^{(n)}$, on one hand, exhibit a lot of nice combinatorial properties: for instance, as will be shown subsequently, they are Sperner, and they manifest the normalized matching property. On the other hand, these posets have some peculiar properties as well: for example, their Hasse diagrams, as graphs, are regular upward, but not regular downward, and their automorphism group has very few elements (see Section 2). (In the sequel we denote arbitrary posets by $\mathcal{P}$.)

The motivation for these investigations involve error-correcting codes with the insertiondeletion distance [10]. In fact, these are collections of $n$-sequences of letters from $\Gamma$, having the property that the length of a subsequence common to any pair of codewords is at most $s<n$. The paper is also related to the so-called longest common subsequence problem and its link to similarity among biological sequences. A good introduction to the latter problem is [7].

As we already mentioned, combinatorics on words has been almost exclusively concerned with the infinite poset $\mathcal{P}^{(\infty)}$, comprising all finite sequences. There are very few results about its finite sub-posets. Here we list those results most relevant to our aims.

Chase studied the number $S_{i}$ of different $i$-subsequences of a given sequence $S$ and proved:

Theorem 1 (Chase, [6]) (i) The $S_{i}, 0 \leq i \leq n$ simultaneously attain their maximum possible values if and only if $S$ is a repeated permutation of the alphabet, that is a sequence with the form of $\left(w_{1} \ldots w_{k}\right) \ldots\left(w_{1} \ldots w_{k}\right) w_{1} \ldots w_{l}$ where $l \equiv n(\bmod k)$ and $w_{1} \ldots w_{k}$ is a fixed permutation of $\Gamma$-or the reverse of such a sequence.

(ii) The numbers $S_{0}, \ldots, S_{|S|}$ are logarithmically concave.

Part (ii) is stated only for the sake of completeness. $|S|$ is used herein to denote the length of sequence $S$. Let $\mathcal{B}_{k, n}$ denote the ideal of $\mathcal{P}^{(n)}$, generated by the maximal element defined in Theorem 1 part (i), and equipped with the derived order.

The poset $\mathcal{B}_{2, n}$ was extensively studied by Burosch, Franke and Röhl in [4]. They determined, among other things, its automorphism group. However, their proof is somewhat 
lengthy. In Section 2 we give a short, new proof for their result and also determine the automorphism group of $\mathcal{P}^{(n)}$.

In [5], Burosch, Gronau and Laborde studied the basic combinatorial properties of the poset $\mathcal{B}_{k, n}$ for general $k$. They determined its Whitney numbers and they conjectured that this poset is Sperner and, even more, it is normal. In this paper we prove the analogous results for $\mathcal{P}^{(n)}$.

\section{Automorphisms}

In this section we study the automorphisms of $\mathcal{P}^{(n)}$ and $\mathcal{B}_{2, n}$. We recall that a bijection on the poset $\mathcal{P}$ is an automorphism if it preserves the order relations. Let $\operatorname{Aut}(\mathcal{P})$ be the automorphism group of the poset $\mathcal{P}$. It is obvious that every permutation $\pi$ of the alphabet $\Gamma$ induces an automorphism $\sigma_{\pi}$ of $\mathcal{P}^{(n)}$, with $\sigma_{\pi}\left(w_{1} w_{2} \ldots w_{t}\right)=\pi\left(w_{1}\right) \pi\left(w_{2}\right) \ldots \pi\left(w_{t}\right)$. Let Sym $_{k}$ denote the automorphism group of $\mathcal{P}^{(n)}$ generated by these $\sigma_{\pi}$ 's.

Let $\rho$ denote the reversal of the order of the letters in all elements of $\mathcal{P}^{(n)}$ (for example $\rho(a b c d)=d c b a)$; then $\rho$ is an automorphism of $\mathcal{P}^{(n)}$ and $\rho^{-1}=\rho$. Let $Z_{2}$ denote the automorphism group of $\mathcal{P}^{(n)}$ generated by $\rho$. It is easy to see that $\rho$ commutes with any other automorphism of $\mathcal{P}^{(n)}$.

In the case of $n=2$, for every (unordered) pair $\{a, b\} \subset \Gamma$, let $\varrho_{a b}$ denote a singleoperation map of $\mathcal{P}^{(2)}$ : the orders of these letters are interchanged whenever they both occur in a 2-sequence. There are $\left(\begin{array}{l}k \\ 2\end{array}\right)$ such maps, and for all distinct (unordered) pairs $\{a, b\}$ and $\{c, d\}$, these automorphisms differ and commute. Therefore, the $\varrho$ 's and an identity constitute a group of automorphisms of $\mathcal{P}^{(2)}$, denoted $Z_{2}^{\left(\begin{array}{c}k \\ 2\end{array}\right)}$. The main result of this section that any automorphism in $\mathcal{P}^{(n)}$ can be derived as the product of one element of $S y m_{k}$ and one of either $Z_{2}$ or $Z_{2}^{\left(\begin{array}{c}k \\ 2\end{array}\right)}$, respectively.

Theorem 2 (i) If $n>2$, then $\operatorname{Aut}\left(\mathcal{P}^{(n)}\right)=S y m_{k} \otimes Z_{2}$;

(ii) if $n=2$, then $\operatorname{Aut}\left(\mathcal{P}^{(n)}\right)=\operatorname{Sym}_{k} \otimes Z_{2}^{\left(\begin{array}{c}k \\ 2\end{array}\right)}$.

The main tool of proof is the following lemma, which is also of intrinsic interest.

Lemma 1 If $3 \leq t$, then every $t$-sequence in $\mathcal{P}_{t}^{(n)}$ is uniquely determined by its set of (distinct) $(t-1)$-subsequences.

Note that if $t=2$, then the statement is obviously false. It is easy to see that the statement is true if our sequence is homogeneous of the form $a a \ldots a$, or if there is no letter occurring twice in the sequence. Thus, henceforth, we assume that our sequence contains a letter $a$ at least twice but that not all letters are the same. Let the sequence $w \in \mathcal{P}_{t}^{(n)}$ contain $s$ copies of the letter $a$ (where $2 \leq s \leq t-1$ ). Then $w$ can be written in the form $w=w_{0} a w_{1} a w_{2} a \ldots w_{s-1} a w_{s}$ where the $w_{j}$ are (possibly empty) a-excluding maximal subsequences. Now any $t-1$ subsequence contains precisely $s-1$ or $s$ copies of $a$. Let $W_{s-1}$ and $W_{s}$ denote the respective sets of distinct $(t-1)$-subsequences. 
We distinguish cases based upon the numbers and lengths of the $w_{j}$ 's. If there are at least two non-empty $w_{j}$ 's in $w$, then one may recognize the $i$-th of these as the longest subsequence occurring in any member of $W_{s}$-between the $i$-th and the $(i+1)$-th copy of $a, 1 \leq i \leq s-1$, and, for $w_{0}$ and $w_{s}$, to the left of the leftmost $a$ or to the right of the rightmost $a$, respectively.

If there is only one non-empty $w_{i}$, denote it $\hat{w}$, and if it is at least two letters long, then one may identify its position among the $a$ 's from any $(t-1)$-subsequence in $W_{s}$. Furthermore, $\hat{w}$ itself may be reconstructed from any $(t-1)$-subsequence belonging to $W_{s-1}$.

Finally, if the only non-empty $w_{i}, \hat{w}$, is a single letter, then consider all the $(t-1)$ subsequences containing $\hat{w}$ in $W_{s-1}$, and count the maximal number of copies of $a$ to the left (and to the right, resp.) of $\hat{w}$. Then $w=a . . a \hat{w} a . . a$, where the respective maximal numbers determine the numbers of $a$ 's on both sides. This proves Lemma 1.

Proof of Theorem 2: It is evident that $\operatorname{Aut}\left(\mathcal{P}^{(n)}\right)$ may only interchange sequences within individual levels of the poset. Take an arbitrary automorphism $\sigma_{0} \in \operatorname{Aut}\left(\mathcal{P}^{(n)}\right)$, and consider its action on $\mathcal{P}_{1}^{(n)}$ (i.e. on the sequences of length 1 ). Thus, this automorphism constitutes a permutation on $\Gamma$. Take its inverse $\pi^{-1}$ on $\Gamma$. This permutation induces an automorphism $\sigma_{\pi^{-1}}$ of the entire poset $\mathcal{P}^{(n)}$. Let $\sigma=\sigma_{0} \sigma_{\pi^{-1}}$. Then, by definition, $\sigma$ is the identity on $\mathcal{P}_{1}^{(n)}$. The same is also true for every homogeneous sequence because the length of any sequence $w \in \mathcal{P}^{(n)}$ corresponds to the length of the longest chain below $w$, which is invariant under any automorphism. Furthermore, any sequence $w \in \mathcal{P}^{(n)}$ and its image under $\sigma$ plainly contain the same letters with the same multiplicities.

First suppose that $n=1$. All 1-sequences are fixed by the automorphism $\sigma$. Now suppose that $n=2$. As before, all 1-sequences and all 2-sequences of form $a a(a \in \Gamma)$ are fixed by the automorphism $\sigma$. There is, however, some freedom with regard to the images of the form $\sigma(a b)$, where $a \neq b \in \Gamma$. (The letters $a, b, c$ will subsequently denote different elements of $\Gamma$.) One may independently prescribe for any (unordered) pair $\{a, b\}$ whether $\sigma(a b)$ should equal $a b$ or $b a$; note that $\sigma(b a)$ will be determined by this prescription. This invokes $Z_{2}^{\left(\begin{array}{c}k \\ 2\end{array}\right)}$ and establishes (ii) of Theorem 2 .

From now on we assume that $n \geq 3$. We may suppose that $\sigma$ fixes the particular twoletter sequence $a b$. (If this is not the case then consider $\sigma \rho$ where $\rho$, as above, reverses all sequences in our poset $\mathcal{P}^{(n)}$.) Since all 1-sequences are fixed it follows that $\sigma(b a)=b a$. Now we can prove

Claim 1 All sequences of type bc are fixed under $\sigma$.

Let $k \geq 3$ and suppose that $\sigma(b c)=c b$. (In the case $k=2$, the claim would be void.) Then $\sigma(b a c)=c b a$ since the order $b a$ is fixed; therefore, $\sigma(a c)=c a$. However, one could not then define $\sigma(b c a)$. This proves the claim.

Now we know that $\sigma$ is the identity on $\mathcal{P}_{1}^{(n)}$ and $\mathcal{P}_{2}^{(n)}$. The application of Lemma 1 establishes the same for $\mathcal{P}_{3}^{(n)}$ and, using induction on $i$, also establishes the same for all $\mathcal{P}_{i}^{(n)}$ where $i=4, \ldots, n$. Theorem 2 is proved. 
Remark: It is natural to enquire whether every $t$-sequence is uniquely determined by its $\ell$-subsequences, for any given $\ell$ with $1<\ell<t$ (and with a trivial lower bound on t). In general, the answer is negative; the sequences $a^{n} b a^{n}$ and $a^{n+1} b a^{n-1}$ with $\ell=\lfloor t / 2\rfloor$ constitute a useful counterexample. On the other hand, the case $\ell=t-1$ yields a positive answer, from Lemma 1 . Thus, the question may be taken to be: For given $t$, what is the minimum value of $\ell$ yielding unique determination?

As we already mentioned in the Introduction, Burosch et al. in [4] studied the poset $\mathcal{B}_{2, n}$, determining its automorphism group. Using our concepts and results, one can straightforwardly reprove their result.

We start with some notation and a remark. In the remainder of this Section, $\mathcal{P}^{(n)}$ denotes the poset with $\Gamma=\{1,2\}$. Furthermore let $\mathcal{B}_{2, n}^{t}$ denote the set of all $t$-sequences in $\mathcal{B}_{2, n}$. We remark that the posets $\mathcal{P}^{(n)}$ and $\mathcal{B}_{2, n}$ are locally isomorphic in the sense that any element $p$ of $\mathcal{B}_{2, n}$, formally, may be found in $\mathcal{P}^{(n)}$, and the generated ideal $\langle p\rangle$ in $\mathcal{B}_{2, n}$ is isomorphic to the ideal $\left\langle p>\right.$ in $\mathcal{P}^{(n)}$. Therefore, it is easy to verify that Lemma 1 is also valid for the poset $\mathcal{B}_{2, n}$. Now we are ready to state and prove the theorem of Burosch et al.

Theorem 3 (i) Consider the poset $\mathcal{B}_{2,2 t+1}$ with $1212 \ldots 121$ as the top element. It has a unique non-identity automorphism, $\rho$, which reverses the order of the letters in every element of the poset.

(ii) Consider the poset $\mathcal{B}_{2,2 t}$ with $1212 \ldots 12$ as the top element. It has a unique non-identity automorphism, $\rho \pi_{2}$, which reverses the order of the letters in every element of the poset and interchanges the 1's and 2's.

Proof: (i) It is easy to check that the map $\rho$ is indeed an automorphism. Furthermore, any automorphism $\sigma$ on $\mathcal{B}_{2,2 t+1}$ is the identity on the homogeneous subsequences of maximal length (since these two subsequences have different lengths: they are $1^{t+1}$ and $2^{t}$.) Therefore $\sigma$ also coincides with the identity on every homogeneous subsequence, including the 1-letter subsequences as well.

Now let $\sigma$ be a particular automorphism. There are two cases: $\sigma(a b)$ could be $a b$ or $b a$. In the first case $\sigma$ is the identity on the two lowest levels; therefore Lemma 1 ensures that $\sigma$ is the identity on the entire poset. In the second case $\sigma \rho$ is the identity on the two lowest levels; therefore (again due to Lemma 1 ) it is just the identity on the entire poset. In this case $\sigma=\rho^{-1}$ which is $\rho$ itself. This proves Theorem 3 (i).

(ii) It is easy to see that the map $\rho \pi_{2}$ is an automorphism and it is own inverse (that is $\left.\rho \pi_{2} \rho \pi_{2}=i d\right)$. We claim that every automorphism should be the identity on the nonhomogeneous two-letter sequences 12 and 21 . The maximum element $1212 \ldots 12$ of our poset has exactly two subsequences (11..112 and 122...22) of length $t+1$ having one 2long non-homogeneous subsequence only (this is 12), but no subsequence of length $t+1$ having 21 as the only non-homogeneous 2-long subsequence; this way 12 and 21 can be distinguished, and they cannot be interchanged by an automorphism.

For an arbitrary automorphism $\sigma$ of $\mathcal{B}_{2,2 t}$ there are two possibilities for the image of $\sigma(11)$. On one hand, if $\sigma(11)=11$, then $\sigma$ is the identity on the two lowest levels, 
and Lemma 1 proves that $\sigma$ is the identity on the entire poset. On the other hand, if $\sigma(11)=22$, then $\sigma(1)=2$ and therefore $\sigma \rho \pi_{2}$ is the identity on the two lowest levels. From Lemma 1 , it is also true for the entire poset. Since $\rho \pi_{2}$ is its own inverse, we have $\sigma=\rho \pi_{2}$, establishing Theorem 3 .

\section{Shades and shadows}

From now on we turn our attention to some purely combinatorial properties of $\mathcal{P}^{(n)}$. In the sequel we consider only finite posets. We recall that our poset $\mathcal{P}^{(n)}$ is a graded one with the sequence length as its rank function. As usual, the rank of any graded poset is the maximum rank of its members (in the case of our poset $\mathcal{P}^{(n)}$, this is $n$ ). Now let $\mathcal{P}$ denote a graded poset with minimum rank 0 , and let $A$ be a set of sequences all having rank $\ell$.

Definition 1 The $i$-shadow $\Delta_{i} A ; 0 \leq i<\ell$; is the subset of the rank- $i$ members of $\mathcal{P}$ whose elements are comparable to one or more element of $A$. If $i=\ell-1$, then we simply say shadow and denote it with $\Delta A$. Analogously, the $i$-shade $\nabla^{i} A ; \ell<i \leq \operatorname{rank}(\mathcal{P})$; is the subset of the rank-i members of $\mathcal{P}$ whose elements are comparable to one or more element of $A$. If $i=\ell+1$, then we simply say shade, denoted by $\nabla A$.

Using these notions, we note that the shadows of elements of the same rank may have different cardinalities. However, our posets behave very regularly upward: any two elements of $\mathcal{P}$ of the same rank have the same $i$-shade cardinality. To prove it we need some further notations. Let $\xi \in \mathcal{P}^{(n)}$ be a fixed sequence $\alpha_{1} \alpha_{2} \cdots \alpha_{|\xi|}$, and let $j$ be an integer such that $|\xi| \leq j \leq n$. Denote $N(j, \xi ; k)$ the number of $j$-sequences, with letters from $\Gamma=\{1,2, \ldots, k\}$, containing the given sequence $\xi$ as a subsequence. Similarly denote $\bar{N}(j, \xi ; k)$ the number of $j$-sequences not containing our fixed $\xi$. In these terms we have

\section{Theorem 4}

$$
\bar{N}(j, \xi ; k)=\sum_{i=0}^{|\xi|-1}\left(\begin{array}{l}
j \\
i
\end{array}\right)(k-1)^{(j-i)} .
$$

Proof: To establish the result, it will suffice to provide an interpretation which verifies each summand. As illustration, we describe the case $i=2$, assuming that $2<|\xi|$. Consider the number of $j$-sequences with the leftmost occurrence of $\alpha_{1}$ at position $g_{1}$, the leftmost subsequent occurrence of $\alpha_{2}$ at position $g_{2}$, and no subsequent occurrence of $\alpha_{3}$, with $1 \leq g_{1}<g_{2} \leq j$. To the left of position $g_{1}$, there are $g_{1}-1$ positions at which any digit from $\Gamma$ except for $\alpha_{1}$ may occur. Between $g_{2}$ and $g_{1}$ there are $g_{2}-g_{1}-1$ positions at which any digit from $\Gamma$ except for $\alpha_{2}$ may occur. To the right of $g_{2}$ there are $n-g_{2}$ positions at which any digit from $\Gamma$ except for $\alpha_{3}$ may occur. Thus, there are a total of $j-2$ positions at which $k-1$ digits may occur, and it follows that $(k-1)^{(j-2)}$ different $j$-sequences are feasible. As there are $\left(\begin{array}{l}j \\ 2\end{array}\right)$ locations for the first occurrence of $\alpha_{1}$ to occur before the first occurrence of $\alpha_{2}$, there are $\left(\begin{array}{l}j \\ 2\end{array}\right)$ of the values $(k-1)^{(j-2)}$. The same argument applies, 
essentially verbatim, to the other summands as well, with $i$ specified digits in place of two. All of the $j$-sequences enumerated in all summands are, clearly, distinct. There are evidently no other $j$-sequences lacking $\xi$ as a subsequence. This proves Theorem 4.

The previous result allows us to establish that any two sequences of the same rank have $i$-shades of the same cardinality.

Corollary 1 Let $\xi$ be a sequence and let $j$ be an integer such that $|\xi| \leq j \leq n$. The number of $j$-sequences containing $\xi$ as a subsequence is

$$
N(j, \xi ; k)=\sum_{i=0}^{j-|\xi|}\left(\begin{array}{l}
j \\
i
\end{array}\right)(k-1)^{i} .
$$

This enumeration follows from (1), using the following "complementation" rule

$$
N(j, \xi ; k)=k^{j}-\bar{N}(j, \xi ; k),
$$

corroborating the result of Levenshtein [12].

\section{Sperner property and LYM property}

In this section we study the basic properties of the antichains of $\mathcal{P}^{(n)}$.

Definition 2 A subset $A$ of the poset $\mathcal{P}$ is called an antichain (or Sperner family) if no two elements of $A$ are comparable.

One of the most fundamental combinatorial properties of a poset is the cardinality of its maximum antichains. The problem originated from the seminal paper of E. Sperner [16], which states that the largest antichain in the Boolean lattice on $n$ elements is formed by the largest family of elements of the same rank (which is the family of all subsets of cardinality $\lfloor n / 2\rfloor$ containing $\left(\begin{array}{c}n \\ \lfloor n / 2\rfloor\end{array}\right)$ elements.) The first theorem of this section addresses this issue for $\mathcal{P}^{(n)}$. While Theorem 8 generalizes the next result, the simplicity of the proof of the special case warrants its separate statement.

The proof is based in the following ideas. A chain in the poset $\mathcal{P}$ is a totally ordered subset of $\mathcal{P}$. A chain cover of $\mathcal{P}$ is a collection of chains, such that every element of $\mathcal{P}$ belongs to at least one chain. It is easy to see that no chain of the cover may contain more then one element from an antichain.

Theorem 5 The cardinality of a maximum antichain of $\mathcal{P}^{(n)}$ is $k^{n}$.

Proof: We may easily construct a chain cover of cardinality $k^{n}$ in $\mathcal{P}^{(n)}$. For every $n$ sequence $\alpha_{1} \alpha_{2} \ldots \alpha_{n}$ we define a chain consisting of the initial segments of that sequence: $\alpha_{1}, \alpha_{1} \alpha_{2}$, and so on through $\alpha_{1} \alpha_{2} \ldots \alpha_{n}$. It is easy to see that these chains really form a chain cover, establishing the upper bound. On the other hand all $n$-sequences clearly form an antichain, proving the statement. 
Let $\mathcal{P}$ be a graded poset with Whitney numbers $\mathcal{W}_{i}$. As was proven independently by Yamamoto [17], Meshalkin [15], Bollobas [2], and Lubell [14], a much stronger form of the Sperner theorem also holds for the antichains in the Boolean algebra $\mathcal{B}_{n}$ on an $n$-element underlying set:

Theorem 6 (BLYM inequality) Let $A$ be an arbitrary antichain in the Boolean algebra $\mathcal{B}_{n}$. Then the following inequality holds:

$$
\sum_{a \in A} \frac{1}{\mathcal{W}_{\operatorname{rank}(a)}} \leq 1
$$

It is a well known fact that all finite, graded, partially ordered sets satisfying an inequality analogous to the one in Theorem 6 (in general it is called the poset LYM inequality) also exhibit the Sperner property. As the following result of Harper and Kleitman (established independently, see $[8,11]$ ) demonstrates, a poset $\mathcal{P}$ satisfies the LYM-inequality if and only if it satisfies the so called normalized matching property (3):

Theorem 7 ([1] Theorem 8.57) Any finite, graded poset $\mathcal{P}$ with Whitney numbers $\mathcal{W}_{0}, \ldots, \mathcal{W}_{n}$ satisfies the LYM-inequality if and only if it satisfies

$$
\frac{|A|}{\mathcal{W}_{i}} \leq \frac{|\nabla A|}{\mathcal{W}_{i+1}}
$$

for all subsets $A$ of the ith level of $\mathcal{P}$, and for all possible $i$.

Our poset $\mathcal{P}^{(n)}$ has Whitney numbers $\mathcal{W}_{i}=k^{i}$. The next result establishes that $\mathcal{P}^{(n)}$ satisfies the normalized matching property:

Theorem 8 The normalized matching property holds for $\mathcal{P}^{(n)}$ : in this case, for any integer $i$ and any $A \subseteq \mathcal{P}_{i}^{(n)}$

$$
k|A| \leq|\nabla A| .
$$

Proof. The proposition is trivially true for $A \subseteq \mathcal{P}_{0}^{(n)}$ because $k=\left|\mathcal{P}_{1}^{(n)}\right|$. Assume, therefore, the truth of the proposition for level $i$, and the inductive proof is completed by demonstrating that this is sufficient to establish the truth of the proposition for level $i+1$. Let $A \subseteq \mathcal{P}_{i+1}^{(n)}$, and partition the elements of $A$ according to their last digit:

$$
\begin{gathered}
A=A_{1} \cup A_{2} \cup \ldots \cup A_{k} ; \\
A_{j}=\left\{s_{j}=\left(\alpha_{1} \cdots \alpha_{i+1}\right) \in A: \alpha_{i+1}=j\right\} ; 1 \leq j \leq k .
\end{gathered}
$$

Let

$$
A_{\mid j} \stackrel{\text { def }}{=}\left\{s_{\mid j}=\left(\alpha_{1} \cdots \alpha_{i}\right):\left(\alpha_{1} \cdots \alpha_{i} j\right) \in A_{j}\right\} ; 1 \leq j \leq k .
$$

We have, 


$$
k|A|=k \sum_{j=1}^{k}\left|A_{j}\right|=k \sum_{j=1}^{k}\left|A_{\mid j}\right| \leq \sum_{j=1}^{k}\left|\nabla A_{\mid j}\right| \leq|\nabla A| .
$$

The central equality arises from the one-to-one correspondence between the members of $A_{j}$ and $A_{\mid j}$, for all $j$. The left inequality is a consequence of the induction hypothesis. The right inequality is not difficult to establish as follows.

First, define a $j$-lifting $L_{j}(B)$ of a set $B \subseteq \mathcal{P}_{i+1}^{(n)}$ :

$$
L_{j}(B)=\left\{L_{j}(b)=\left(\alpha_{1} \alpha_{2} \cdots \alpha_{i+1} j\right): b=\left(\alpha_{1} \alpha_{2} \cdots \alpha_{i+1}\right) \in B\right\} ; 1 \leq j \leq k .
$$

That is, a $j$-lifting the sequences of $B$ suffixes them with the digit $j$. If a sequence covering $s_{\mid j}=\left(\alpha_{1} \cdots \alpha_{i}\right) \in A_{\mid j}$ is suffixed by $j$, then a sequence covering $s_{j}=\left(\alpha_{1} \cdots \alpha_{i} j\right) \in A_{j}$ is, obtained. Therefore, $\sum_{j=1}^{k}\left|\nabla A_{\mid j}\right|=\sum_{j=1}^{k}\left|L_{j}\left(\nabla A_{\mid j}\right)\right| \leq|\nabla A|$. This completes the proof of Theorem 8.

As we mentioned earlier, this result implies the following one:

Corollary 2 Our poset $\mathcal{P}^{(n)}$ satisfies the $L Y M$-inequality: let $A \subset \mathcal{P}^{(n)}$ be an antichain. Then

$$
\sum_{a \in A} \frac{1}{k^{\operatorname{rank}(a)}} \leq 1
$$

Furthermore, Theorem 5 is also a consequence of Theorem 8 .

The authors are indebted to the unknown referees. One of them pointed out several insufficiently clear points in our manuscript. The other referee formulated the problem described in the Remark after Theorem 2.

\section{References}

[1] M. Aigner: Combinatorial Theory, Springer-Verlag, Berlin, 1979, p. 428.

[2] B. Bollobas: On generalized graphs, Acta Math. Acad. Sci. Hung. 16 (1965), 447-452.

[3] E. J. Borowski and J. M. Borwein: The HarperCollins Dictionary of Mathematics, HarperCollins Publishers, New York, 1991.

[4] G. Burosch, U. Franke, S. Röhl: Über Ordnungen von Binärworten, Rostock. Math. Kolloq. 39 (1990), 53-64.

[5] G. Burosch, H-D. Gronau, J-M. Laborde: On posets of $m$-ary words, Discrete Math. 152 (1996), 69-91.

[6] P.J. Chase: Subsequence numbers and logarithmic concavity, Discrete Math. 16 (1976), 123-140.

[7] D. Gusfield: Algorithms on Strings, Trees and Sequences, Cambridge University Press, Cambridge, 1997. 
[8] L.H. Harper: The morphology of partially ordered sets, J. Comb. Theory (A) 17 (1974),44-59.

[9] G. Higman: Ordering by divisibility in abstract algebra, Proc. London Math. Soc. 2 (1953), 326-336.

[10] H.D.L. Hollmann: A relation between Levenshtein-type distances and insertion-anddeletion correcting capabilities of codes, IEEE Trans. on Information Theory $\mathbf{3 9}$ (1993), 1424-1427.

[11] D.J. Kleitman: On an extremal property of antichains in partially orders. The LYM property and some of its implications and applications, Combinatorics (Hall \& van Lints, eds.) Math. Centre Tracts 56 Amsterdam, (1974) 77-90.

[12] V.I. Levenshtein: On perfect codes in deletion and insertion metric, Discrete Math. Appl. 2 (1992), 241-258.

[13] M. Lothaire: Combinatorics on words, Cambridge University Press, Cambridge, 1997.

[14] D. Lubell: A short proof of Sperner's lemma, J. Combin. Theory, 1 (1966), 299.

[15] L.D. Meshalkin: A generalization of Sperner's theorem on the number of subsets of a finite set, (Russian) Teor. Verojatnost. i Primenen 8 (1963), 219-220.

[16] E. Sperner: Ein Satz über Untermengen einer endlichen Menge, Math. Z. 27 (1928), $544-548$.

[17] K. Yamamoto: Logarithmic order of free distributive lattice, J. Math. Soc. Japan 6 (1954), 343-353. 